\title{
Backward Free Convection Boundary Layers in Porous Media
}

\author{
E. MAGYARI* and B. KELLER \\ Chair of Physics of Buildings, Institute of Building Technology, Swiss Federal Institute \\ of Technology (ETH) Zürich, Wolfgand-Pauli-Str.1, CH-8093 Zürich, Switzerland
}

(Received: 30 September 2002)

\begin{abstract}
The well known steady free convection forward boundary layer (FBL) flows ascending over a heated upwards projecting semi-infinite flat plate embedded in a fluid saturated porous medium are compared in this paper to their less well known backward (BBL) counterparts descending over a cooled (also upwards projecting!) semi-infinite flat plate. The circumstance that the definite edge of the plate $(x=0)$ in the former case is a leading edge and in the latter one a trailing edge, leads to substantially different mathematical and physical features of the FBL and BBL flows, respectively. The paper considers under this aspect the case of similar flows corresponding to surface temperature distributions which are power-law functions of the distance $x$ from the definite edge. For permeable plates the effect of an adequate lateral suction and injection of the fluid is also taken into account. The detailed investigation, however, is restricted to the particular values $m=+1$ and $m=-1 / 3$ of the power-law exponent $m$, where both FBL and BBL solutions are available in exact analytic form. For each of these values, both exponentially and algebraically decaying BBL solutions were found. In addition, the existence of an exact algebraic BBL solution valid for any value of $m$ is reported.
\end{abstract}

Key words: free convection, vertical surface, backward boundary-layer, power-law similarity, exact solutions, algebraic decay, suction, injection.

\section{Introduction}

The term 'backward boundary layer' (BBL) as a counterpart of a usual, or 'forward boundary layer' (FBL) has been introduced into the fluid mechanics by Goldstein by the words: "In a backward boundary layer the fluid has, in the mathematical model, been flowing along a solid wall through an infinite distance" (Goldstein, 1965).

For a BBL past a semi-infinite plate, this means that the leading edge recedes to an indefinite station infinitely far upstream, while the definite edge of the plate becomes a trailing edge (Figure 1( $\mathrm{a}$ and $\mathrm{b})$ ). As a consequence, in the BBL the fluid has lost any memory of the perturbations introduced by the leading edge. This circumstance makes the BBL's to an interesting research object. Moreover, although in general not mentioned explicitly, the so called opposing case of a mixed convection flow over a cooled upward projecting semi-infinite plate, is nothing than

\footnotetext{
${ }^{*}$ Author for correspondence.
} 


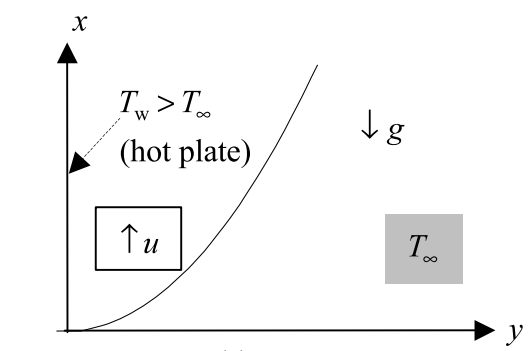

0

(a) FBL

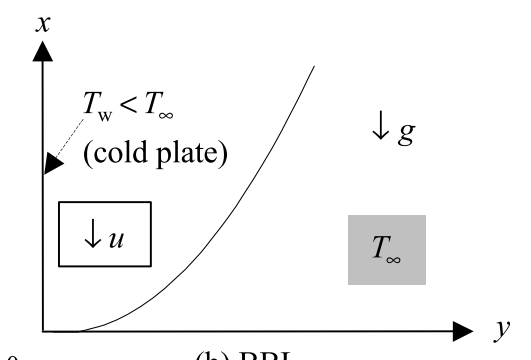

(b) BBL

Figure 1. Flow domain of a free convection FBL ascending along a semi-infinite heated plate (a) and of its backward counterpart (BBL) descending along a cooled plate (b), respectively. In the first case, the definite edge of the plate $(x=0)$ is a leading edge while in the second case, $x=0$ becomes a trailing edge.

the competition a forward forced convection flow with a backward free convection flow. This fact even enhances the research interest in the BBL's.

Obviously, all the above mentioned features are encountered both in the case of viscous flow of clear fluids as well as in the viscous fluid flow in saturated porous media.

In Goldstein's pioneering paper (Goldstein, 1965) the BBL flow of a clear viscous fluid in a converging passage has been considered. The fluid enters at infinity with infinitely slow velocity, and moves in the converging passage towards the intersection of the walls, which is taken as origin $(x=0)$. Assuming a mainstream velocity of the form $U \sim-x^{m}$, Goldstein has shown that for $-1<m<0$ algebraically decaying BBL solutions could exist. He concluded, however, that such solutions were unacceptable as they could not be matched with the outer expansion over a finite portion of the $x$ axis (since this matching would rather require an exponential decay). Subsequently, Brown and Stewartson (1965) and later Merkin (1978) have amended Goldstein's conclusion in that they showed that algebraic behavior can be admitted only at singular points in the flow field, such as sources, sinks or points of separation, but along portions of finite length, potential flow and algebraic decay are mutually exclusive. It seemed therefore, that in the theory of plane boundary layers the algebraic decay possesses a marginal significance only. An important milestone in changing this view is marked by the papers of Kuiken published in the early eighties (Kuiken, 1981a, b, 1983). Kuiken has shown that the BBL flows without external stream as those induced by continuous surfaces which are stretched in quiescent fluids (and disappear in a narrow slot) can actually decay algebraically along wall portions which are not vanishingly small (Kuiken, 1981a). This holds also for the free convection BBL flow due to the cooling of a low-heat-resistance sheet that moves slowly in the downward direction (Kuiken, 1981b). By introducing a shifted longitudinal coordinate, Kuiken succeeded to find similarity solutions to this free convection problem. Subsequently, Khan and Stewartson (1984) have extended Kuiken' similarity solution by embedding it into the full solution of a certain system of partial differential equations. They have 
shown that Kuiken's solution gives a remarkably accurate result for the heat transfer coefficient and the skin friction. However, the displacement thickness differs significantly from the similarity prediction almost everywhere (see also Pop and Ingham, 2001, Chapter 8). Kuiken has also shown that in the case of a heated downward-projecting semi-infinite fixed slab of uniform thickness a similar free convection BBL solution can be found (numerically) by assuming that the temperature of the plate varies in proportion to the inverse of the seventh power of the (shifted) longitudinal coordinate (Kuiken, 1983).

The present paper considers the problem of the free convection BBL flows from a semi-infinite vertical plate with a power law temperature distribution by assuming that the plate is embedded in a fluid saturated porous medium. The main focus of the paper are the particular cases $m=+1$ and $m=-1 / 3$ of the power-law exponent $m$, where both exponentially and algebraically decaying BBL solutions will be presented in an explicit analytic form. Also the existence of an exact algebraic BBL solution valid for any value of $m$ is reported. In addition, for $m=-1 / 3$ a new class of algebraically decaying exact FBL solutions will be described.

\section{Basic Considerations}

Following Nield and Bejan (1999) we write the mass, momentum and energy conservation equations of a Darcy-Boussinesq free convection boundary-layer flow over a semi-infinite vertical flat plate adjacent to a saturated porous medium in the form:

$$
\begin{aligned}
& u_{x}+v_{y}=0 \\
& u=\frac{g \beta K}{v}\left(T-T_{\infty}\right) \\
& u T_{x}+v T_{y}=\alpha T_{y y}
\end{aligned}
$$

Here $x \geqslant 0$ and $y \geqslant 0$ are the Cartesian coordinates along and normal to the plate, respectively, $u$ and $v$ are the velocity components along $x$ and $y$ axes, $T=(x, y)$ is the local and $T_{\infty}$ the ambient temperature of the fluid, $K$ is the permeability of the porous medium, $g$ is the acceleration due to gravity, $c_{\mathrm{p}}$ is the specific heat at constant pressure, $\alpha, \beta$ and $v=\mu / \rho$ are the effective thermal diffusivity, thermal expansion coefficient and kinematic viscosity, respectively, and the subscripts $x$ and $y$ indicate partial derivatives. The $x$-axis, with its origin on the finite edge of the plate, is directed vertically upwards, (Figure 1(a and b)). The plate will be considered as being impermeable or permeable. In the latter case the effect of a lateral suction or injection of the fluid will also be examined. In this way, the boundary conditions accompanying Equations (1)-(3) are:

$$
\begin{aligned}
& T=T_{\mathrm{w}}(x), \quad v=v_{\mathrm{w}}(x) \quad \text { on } y=0 \\
& T \rightarrow T_{\infty} \quad \text { as } \quad y \rightarrow \infty:
\end{aligned}
$$

where the wall functions $T_{\mathrm{w}}(x)$ and $v_{\mathrm{w}}(x)$ are prescribed. 
Concerning the wall temperature distribution $T(x, 0)=T_{\mathrm{w}}(x)$ we assume a power-law dependence on $x$,

$$
T_{\mathrm{w}}(x)=T_{\infty}+s \cdot T_{0} \cdot\left(\frac{x}{L}\right)^{m}, \quad T_{0}>0, \quad s= \pm 1
$$

where $L$ is a reference length (which for $m \neq 0$ represents the distance $x$ at which $T_{\mathrm{w}}(x)$ takes a prescribed value $\left.T_{\mathrm{w}}(L)=T_{\infty}+s \cdot T_{0}\right)$. Thus the wall temperature is either higher or lower than the ambient temperature of the fluid $T_{\infty}$ for any $x \geqslant 0$. In this sense we speak about a 'heated' $(s=+1)$ or a 'cooled' $(s=-1)$ plate, respectively (Figure 1(a and b)).

Owing to Equation (2), the velocity field can be decoupled from the temperature field and, besides the continuity equation (1), it satisfies the energy equation

$$
u u_{x}+v u_{y}=\alpha u_{y y}
$$

along with the boundary conditions

$$
\begin{aligned}
& u=s \frac{\alpha}{L} R a\left(\frac{x}{L}\right)^{m}, \quad v=v_{\mathrm{w}}(x) \quad \text { on } y=0 \\
& u \rightarrow 0 \quad \text { as } y \rightarrow \infty
\end{aligned}
$$

where $R a$ denotes the Darcy-Rayleigh number, $R a=g \beta K T_{0} L /(v \alpha)$.

The sign $s$ affects only the wall conditions, showing that we are faced here, as expected, either with an ascending $(s=+1)$ or a descending $(s=-1)$ boundary layer flow, respectively. The former is the familiar FBL and the latter the less familiar BBL, respectively (Figure 1(a and b)). In the context of porous media, the forward free convection boundary layer flows have been investigated in the past few decades very intensively (for a recent overview, see e.g. Nield and Bejan, 1999 and Pop and Ingham, 2001). However, as far as we are aware, the backward ones were not considered. Their investigation is the main issue of the present paper.

\section{Similarity Equations}

It is well known that the power law wall temperature distribution (5) allows for similarity solutions (see e.g. Pop and Ingham, 2001). The corresponding governing equations can easily be obtained by introducing the stream function $\psi$ and the similarity variable $\eta$ according to the definitions

$$
\psi=s \alpha \sqrt{R a} \cdot X^{(m+1) / 2} \cdot f(\eta), \quad \eta=\sqrt{R a} \cdot X^{(m-1) / 2} \cdot Y
$$

where $X=x / L$ and $Y=y / L$ are new dimensionless coordinates. In this way the solution of our problem results as follows:

$$
\begin{aligned}
& u \equiv \psi_{y}=s \frac{\alpha}{L} R a \cdot X^{m} \cdot f^{\prime}(\eta) \\
& v \equiv-\psi_{x}=-s \frac{\alpha}{L} \sqrt{R a} \cdot X^{(m-1) / 2} \cdot\left[\frac{m+1}{2} f(\eta)+\frac{m-1}{2} \eta \cdot f^{\prime}(\eta)\right] \\
& T=T_{\infty}+s T_{0} \cdot X^{m} \cdot \vartheta(\eta), \quad \vartheta(\eta) \equiv f^{\prime}(\eta) .
\end{aligned}
$$


Here the dimensionless stream function $f$ satisfies the ordinary differential equation

$$
f^{\prime \prime \prime}+s \cdot\left(\frac{1}{2}(m+1) f f^{\prime \prime}-m f^{\prime 2}\right)=0
$$

along with the boundary conditions

$$
f(0)=f_{\mathrm{w}}, \quad f^{\prime}(0)=1, \quad f^{\prime}(\infty)=0
$$

and the primes denote derivatives with respect to $\eta$. The dimensionless suction/ injection velocity $f_{\mathrm{w}}$ is specified by Equation (10) as follows:

$$
v_{\mathrm{w}}(x)=-s \frac{\alpha(m+1) \sqrt{R a} f_{\mathrm{w}}}{2 L} X^{(m-1) / 2} .
$$

Hence, for $m \neq-1$ the value $f_{\mathrm{w}}=0$ corresponds to an impermeable plate $\left(v_{\mathrm{w}}=0\right)$ and the non-vanishing values of $f_{\mathrm{w}}$ are associated with a permeable plate $\left(v_{\mathrm{w}} \neq 0\right)$. In the latter case similarity solutions exist only if a lateral suction or injection is applied according to Equation (14).

In the special case $m=-1$ the boundary value problem $(12,13)$ does not admit FBL solutions, neither for a vanishing nor for a non-vanishing $f_{\mathrm{w}}$. It admits however an elementary BBL solution which will be discussed in Section 4 below.

Therefore, the velocity and temperature fields are fully determined for any $m \neq-1$ if the dimensionless stream function $f=f(\eta)$ is known (in the case $m=-1$ it is sufficient to specify its first derivative $f^{\prime}(\eta)$, only). In addition to the velocity and temperature fields, our interest will be directed on the heat transfer coefficient

$$
H \equiv \frac{N u_{x}}{\sqrt{R a_{x}}}=-s \cdot f^{\prime \prime}(0)=-s \cdot \vartheta^{\prime}(0)
$$

where the local Nusselt and Rayleigh numbers are defined as

$$
N u_{x}=\frac{q_{\mathrm{w}}(x) \cdot x}{k \cdot\left|T_{\mathrm{w}}(x)-T_{0}\right|}, \quad R a_{x}=R a \cdot X^{m+1}
$$

and the wall heat flux is given by

$$
q_{\mathrm{w}}(x)=-k \frac{\partial T}{\partial y}(x, 0)=-s \frac{k T_{0}}{L} \sqrt{R a} \cdot X^{(3 m-1) / 2} \cdot f^{\prime \prime}(0) .
$$

Notice, that due to the inclusion of $s$ in the stream function (8) and due to the definition of the dimensionless suction/injection velocity $f_{\mathrm{w}}$ according to Equation (14), the sign $s$ which distinguishes the FBL's from the BBL's has been transferred from the boundary conditions of the problem $(6,7)$ into the basic differential equation of the problem $(12,13)$.

The next Section of the paper is devoted to the 'universal' BBL solution of the problem $(12,13)$. Then, in Sections 5 and 6 we examine the BBL flows descending 
over the cooled plate of Figure 1(b) and compare them to their forward counterparts, ascending over the heated plate of Figure 1(a), both corresponding to the values 1 and $-1 / 3$ of the temperature exponent $m$, respectively.

\section{The 'Universal' BBL Solution}

It is easy to check that

$$
f(\eta)=-\frac{6}{\eta+\sqrt{6}}, \quad \eta=\sqrt{R a} \cdot X^{(m-1) / 2} \cdot Y
$$

is a universal BBL solution of the problem $(12,13)$ for any $m$. Under 'universal solution' we mean that (17) holds for any $m$. Thus, for $m \neq-1$ the universal BBL is always formed over a permeable plate $\left(v_{\mathrm{w}} \neq 0\right)$ and it can be realized only if a lateral suction or injection with $f_{\mathrm{w}}=-\sqrt{6}$ is applied. Equation (14) shows that for $s=-1, f_{\mathrm{w}}=-\sqrt{6}$ means suction $\left(v_{\mathrm{w}}<0\right)$ if $m>-1$ and injection $\left(v_{\mathrm{w}}>0\right)$ of the fluid if $m<-1$, respectively. The velocity and temperature fields corresponding to the dimensionless stream function (17) are:

$$
\begin{aligned}
& u=-\frac{\alpha}{L} R a \cdot X^{m} \frac{6}{(\eta+\sqrt{6})^{2}} \\
& v=-\frac{3 \alpha}{L} \sqrt{R a} \cdot X^{(m-1) / 2} \frac{2 \eta+(m+1) \sqrt{6}}{(\eta+\sqrt{6})^{2}} \\
& T=T_{\infty}-T_{0} X^{m} \frac{6}{(\eta+\sqrt{6})^{2}} .
\end{aligned}
$$

The entrainment/displacement velocity of this flow is zero, $v(x, \infty)=0$. The heat transfer coefficient (15) corresponding to the temperature field (20) is

$$
H=\frac{N u_{x}}{\sqrt{R a_{x}}}=-\sqrt{\frac{2}{3}} \cong-0.81649 .
$$

As expected, $H$ is always negative.

The universal BBL solution (17) holds also for $m=-1$. Equation (14) shows that the corresponding BBL is formed over an impermeable surface $\left(v_{\mathrm{w}}=0\right)$, regardless the value of $f_{\mathrm{w}}$. Since in this case all the physical fields (9)-(11) depend only on $f^{\prime}(\eta)$ but not on $f(\eta)$, the BBL solution (17) is determined for $m=-1$ only to an additive constant (such that $f(0)=f_{\mathrm{w}}=-\sqrt{6}$ has no any physical significance in this case).

It is worth mentioning here that in the FBL-case $(s=+1)$, no 'universal' boundary layer solution comparable with (17) is known. 


\section{BBL Versus FBL Flows for $m=+1$}

For a linear surface temperature distribution, $m=+1$, elementary analytical solutions exist both in FBL as well as in BBL case. The former are well known, both in the present context of porous media (see e.g. Magyari and Keller, 2000a), as well as in the mathematically analogous context of boundary layer flows induced by continuous stretching surfaces (see e.g. Banks, 1983; Magyari and Keller, 2000b). However, their backward counterparts, although of a nearly identical form, were not yet been reported in the literature.

It is easy to show that for $m=+1$ the FBL and BBL solutions of the problem $(12,13)$ are given by

$$
f(\eta)=H-\frac{\mathrm{e}^{-s H \eta}}{s H}, \quad f^{\prime}(\eta)=\mathrm{e}^{-s H \eta}, \quad \eta=\sqrt{R a} \cdot Y
$$

for $s=+1$ and $s=-1$, respectively. Here the heat transfer coefficient $H=-s$. $f^{\prime \prime}(0)=-s \cdot \vartheta^{\prime}(0)$ is obtained as the positive or negative root(s) of the quadratic equation $H^{2}-f_{\mathrm{w}} H-s=0$ for $s=+1$ and $s=-1$, respectively. Thus

$$
H \equiv H_{\mathrm{F}}=\frac{1}{2}\left(f_{\mathrm{w}}+\sqrt{f_{\mathrm{w}}^{2}+4}\right)>0, \quad-\infty<f_{\mathrm{w}}<+\infty
$$

for the FBL flows and

$$
H \equiv H_{ \pm}=\frac{1}{2}\left(f_{\mathrm{w}} \pm \sqrt{f_{\mathrm{w}}^{2}-4}\right)<0, \quad f_{\mathrm{w}} \leqslant-2
$$

for the BBL flows, respectively, that is:

$$
H \equiv \frac{N u_{x}}{\sqrt{R a_{x}}}=\left\{\begin{array}{ll}
H_{\mathrm{F}} & \text { for the FBL's } \\
H_{+} \text {and } H_{-} & \text {for the BBL's }
\end{array} .\right.
$$

As expected, $H$ is positive in the FBL case and negative in the BBL case, respectively. In both cases the fluid is entrained from the outer domain with a constant velocity, $v(x, \infty)=-s(\alpha / L) \sqrt{R a} \cdot H<0$. While the FBL's exist both on an impermeable $\left(f_{\mathrm{w}}=0\right)$ and a permeable $\left(f_{\mathrm{w}} \neq 0\right)$ plate in the presence of a lateral suction $\left(f_{\mathrm{w}}>0\right)$ or injection $\left(f_{\mathrm{w}}<0\right)$ of the fluid, the BBL's, according to Equation (24), are only possible if a sufficiently strong suction $\left(f_{\mathrm{w}} \leqslant-2\right)$ is applied. Moreover, whereas the FBL solutions are unique, the BBL solutions (22) are dual solutions for any $f_{\mathrm{w}} \leqslant-2$. The two solution branches corresponding to $H$ 's given by Equation (24) bifurcate at the value -2 of the suction parameter $f_{\mathrm{w}}$ (Figure 2$)$. The point $(-\sqrt{6},-\sqrt{2 / 3})$ of the plane $\left(f_{\mathrm{w}}, H\right)$ corresponding to the universal BBL solution (17) lies on the straight line which connects the solution branches $H_{ \pm}$at $f_{\mathrm{w}}=-\sqrt{6}$. Numerical calculations show that all the other points of this straight line also correspond to BBL-solutions of the boundary value problem $(12,13)$. Moreover, this holds for any point inside the domain of the plane $\left(f_{\mathrm{w}}, H\right)$ delimited by the curves $-H_{+}$and $-H_{-}$. Therefore, this domain together with its border curves $H_{+}$and $H_{-}$, represents the existence domain of the similar BBL solutions of the boundary value problem $(12,13)$ for $m=1$ and the 


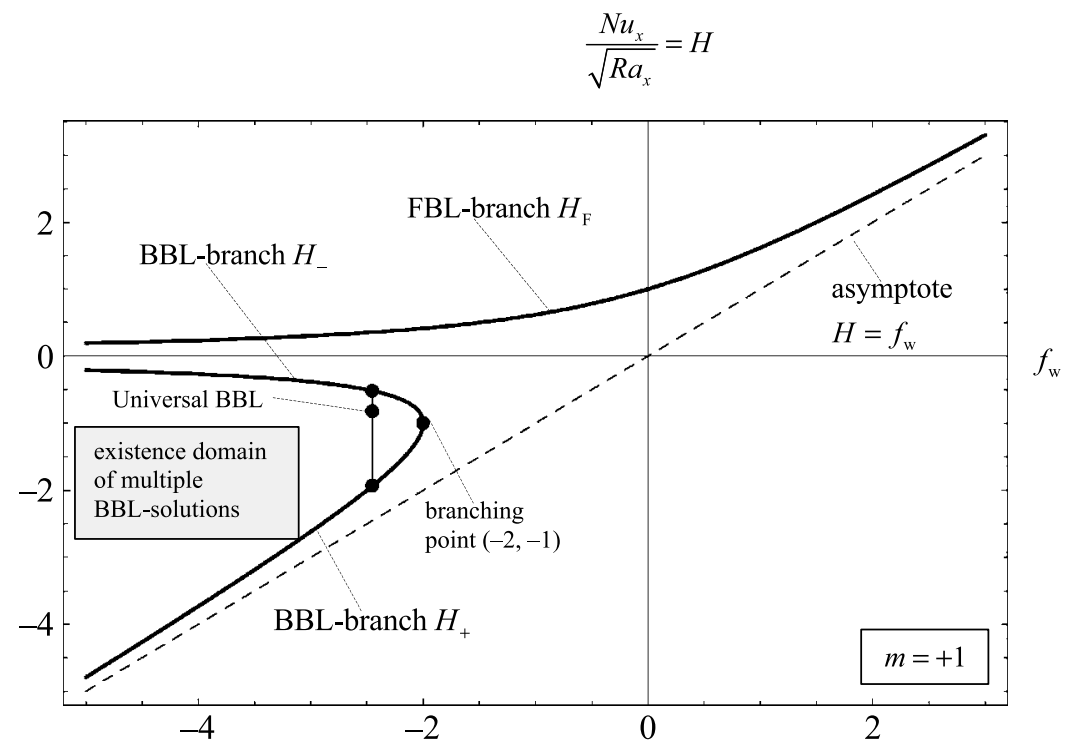

Figure 2. Existence domains of the unique FBL and of the multiple BBL solutions for $m=1$.

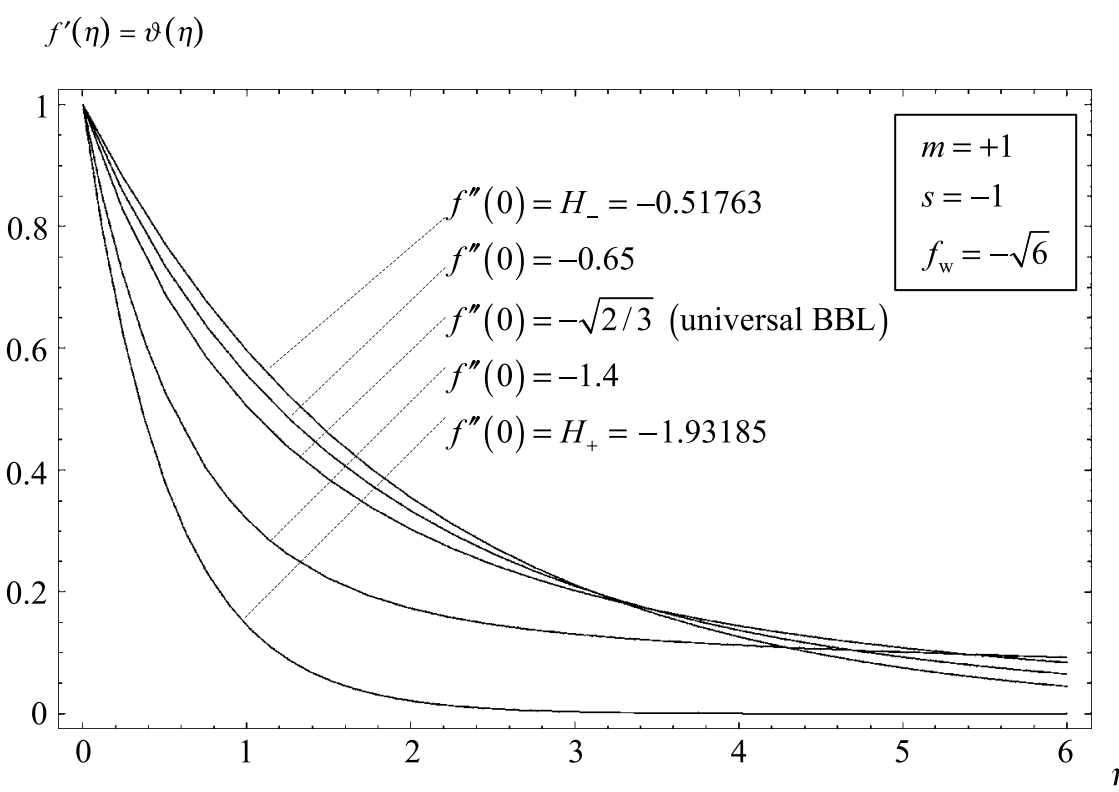

Figure 3. Shape of the dimensionless downstream velocity and temperature profiles $f^{\prime}(\eta)=\vartheta(\eta)$ of the multiple BBL's for $m=1$ and $f_{\mathrm{w}}=-\sqrt{6}$, and five different values of $f^{\prime \prime}(0)=H\left(f_{\mathrm{W}}\right)$.

point $\left(f_{\mathrm{w}}, H\right)=(-2,-1)$ is the branching point of these multiple BBL solutions. All these results are collected in Figure 2, where the heat transfer coefficient $H$ is plotted versus $f_{\mathrm{w}}$. As an illustration, in Figure 3 the shape of the dimensionless 
downstream velocity and temperature profiles $f^{\prime}(\eta)=\vartheta(\eta)$ is shown for $f_{\mathrm{w}}=$ $-\sqrt{6}$ and different values of $H$ lying between the corresponding ordinates $H_{-}=$ -0.51763 and $H_{+}=-1.93185$ of the border curves $H_{ \pm}$, respectively. It is worth underlying here that the BBL solutions (22) corresponding to the border curves $H_{ \pm}$ are exponentially decaying functions. At $f_{\mathrm{w}}=-\sqrt{6}$, they represent limiting cases of a family of BBL solutions which go over smoothly in the universal algebraic BBL solution (17) as $H$ changes continuously from $H_{-}=-0.51763$ and $H_{+}=$ -1.93185 to $H=-\sqrt{2 / 3}$, respectively. This result is quite surprising.

\section{BBL Versus FBL Flows for $m=-1 / 3$}

We first notice that our basic equation (12) can be transcribed in the form

$$
\frac{d^{2}}{d \eta^{2}}\left[f^{\prime}+\frac{s}{4}(m+1) f^{2}\right]-\frac{s}{2}(3 m+1) f^{\prime 2}=0 .
$$

Thus, for $m=-1 / 3$ we obtain after two elementary integrations the Riccati equation

$$
f^{\prime}+\frac{1}{6} s f^{2}=C_{1} \eta+C_{2}
$$

For the integration constants $C_{1}$ and $C_{2}$, the first two boundary conditions (13) yield

$$
C_{1}=f^{\prime \prime}(0)+\frac{1}{3} s f_{\mathrm{w}}, \quad C_{2}=1+\frac{1}{6} s f_{\mathrm{w}}^{2} .
$$

Hence, for a given value of $f_{\mathrm{w}}$ (and a specified $s$ ) those values of $f^{\prime \prime}(0)$, that is, of $C_{1}$, are sought for which Equation (27) admits solutions which satisfy the first and the third boundary conditions (13). Obviously, an additional physical requirement is that the dimensionless temperature field $\vartheta(\eta)=f^{\prime}(\eta)$ is a bounded and positive function of $\eta$ for any $0 \leqslant \eta<\infty$.

Corresponding to the vanishing or non-vanishing value of $C_{1}$, two main classes of solutions must be distinguished.

\subsection{CASE $c_{1}=0$}

In this case we have

$$
f^{\prime \prime}(0)=\vartheta^{\prime}(0)=-\frac{1}{3} f_{\mathrm{w}}
$$

and the third boundary condition (13) yields

$$
f_{\infty}^{2}=f_{\mathrm{w}}^{2}+6 s, \quad f_{\infty} \equiv \lim _{\eta \rightarrow \infty} f(\eta)
$$

such that

$$
f^{\prime}=\frac{1}{6} s\left(f_{\infty}^{2}-f^{2}\right) .
$$


There occur again two distinct cases, corresponding to a vanishing or to a nonvanishing $f_{\infty}$, respectively. The case $f_{\infty}=0$ implies $f_{\mathrm{w}}^{2}=-6 s$ and thus, it can only be realized as a BBL $(s=-1)$ over a permeable surface. The corresponding solution of Equation (31) is precisely the universal solution (17) with $f_{\mathrm{w}}=-\sqrt{6}$ (suction).

The FBL solutions corresponding to $f_{\infty} \neq 0$ (and $C_{1}=0$ ) are well known and read (Magyari and Keller, 2000a, b):

$$
\begin{aligned}
& f_{+}(\eta)=w_{+} \tanh \left(\frac{w_{+}}{6} \eta+\frac{1}{2} \ln \frac{w_{+}+f_{\mathrm{w}}}{w_{+}-f_{\mathrm{w}}}\right), \\
& w_{+}=f_{+}(\infty)=\sqrt{f_{\mathrm{w}}^{2}+6}, \quad-\infty<f_{\mathrm{w}}<+\infty \\
& f_{+}^{\prime}(\eta)=\vartheta_{+}(\eta)=\frac{w_{+}^{2}}{6} \cosh ^{-2}\left(\frac{w_{+}}{6} \eta+\frac{1}{2} \ln \frac{w_{+}+f_{\mathrm{w}}}{w_{+}-f_{\mathrm{w}}}\right), \\
& f_{+}^{\prime \prime}(0)=-\frac{1}{3} f_{\mathrm{w}} .
\end{aligned}
$$

The new BBL counterparts of (32), (33) are

$$
\begin{aligned}
& f_{-}(\eta)=-\frac{w_{-}}{\tanh \left(\left(w_{-} / 6\right) \eta+(1 / 2) \ln \left(f_{\mathrm{w}}-w_{-}\right) /\left(f_{\mathrm{w}}+w_{-}\right)\right)}, \\
& w_{-}=-f_{-}(\infty)=\sqrt{f_{\mathrm{w}}^{2}-6}, \quad f_{\mathrm{w}} \leqslant-\sqrt{6} \\
& f_{-}^{\prime}(\eta)=\vartheta_{-}(\eta)=\frac{w_{-}^{2}}{6} \sinh ^{-2}\left(\frac{w_{-}}{6} \eta+\frac{1}{2} \ln \frac{f_{\mathrm{w}}-w_{-}}{f_{\mathrm{w}}+w_{-}}\right), \\
& f_{-}^{\prime \prime}(0)=+\frac{1}{3} f_{\mathrm{w}} .
\end{aligned}
$$

We see therefore that in the forward case the temperature profile (33) can be realized on an impermeable plate $\left(f_{\mathrm{w}}=0\right)$, as well as on a permeable plate in the presence of both a lateral suction $\left(f_{\mathrm{w}}>0\right)$ or injection $\left(f_{\mathrm{w}}<0\right)$ of the fluid. The BBL's (35) on the other hand, only exist if a sufficiently strong suction with $f_{\mathrm{w}} \leqslant-\sqrt{6}$ is applied. In these flows, the fluid is entrained from the outer domain with velocities $v_{ \pm}(x, \infty)=-(\alpha / 3 L) \sqrt{R a \cdot\left(f_{\mathrm{w}}^{2} \pm 6\right)} \cdot X^{-2 / 3}<0$, respectively. It can be shown that in the limiting case $f_{\mathrm{w}} \rightarrow-\sqrt{6}$ the exponential BBL solution (34) goes over precisely in the universal algebraic BBL solution (17). The heat transfer coefficient (15) is given in case $C_{1}=0$ for both the FBL's and BBL's by the same expression

$$
H=-s \cdot f^{\prime \prime}(0)=\frac{1}{3} f_{\mathrm{w}}, \quad C_{1}=0 .
$$

Therefore, the heat transfer coefficient (36) of the above FBL's and BBL's is represented in the plane $\left(f_{\mathrm{w}}, H\right)$ of Figure 4 by the same straight line $f_{\mathrm{w}} / 3$ with 


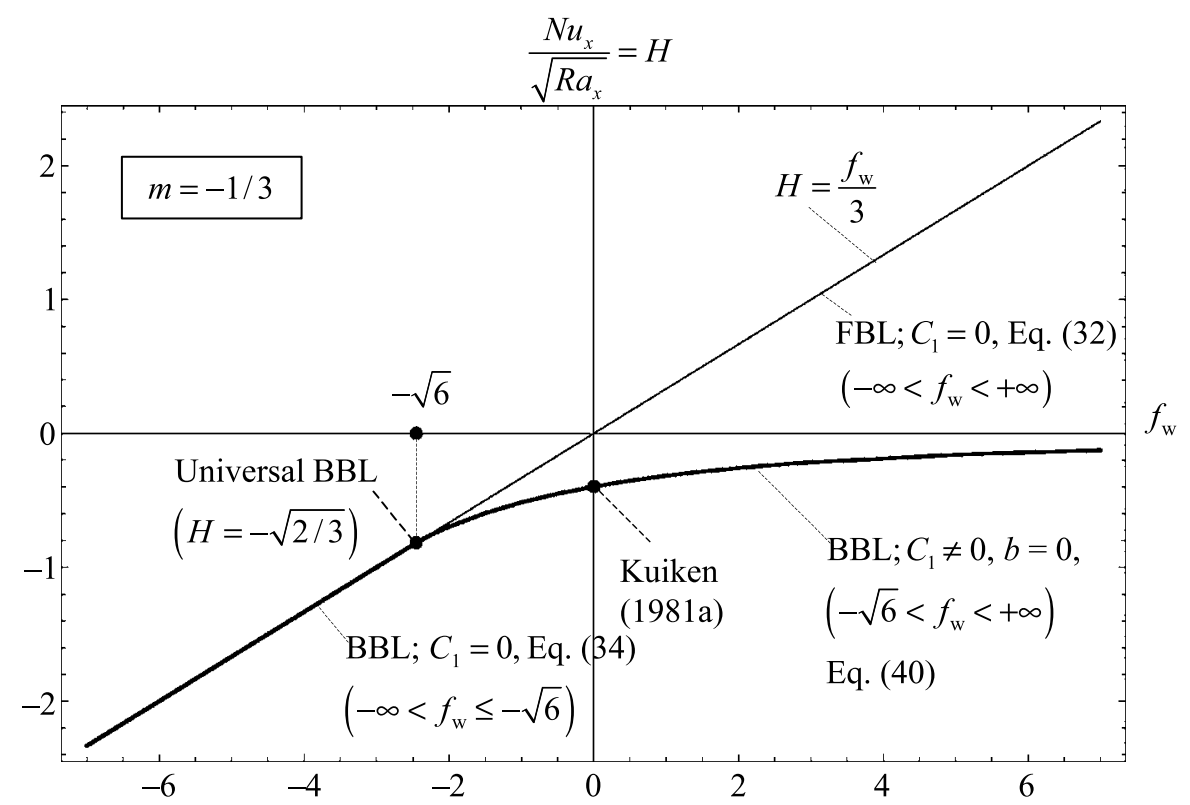

Figure 4. Existence domains of the FBL and BBL solutions for $m=-1 / 3$.

the essential difference that while the domain of existence of the FBL's covers the whole $f_{\mathrm{w}}$-axis, that of the BBL's is restricted to the range $f_{\mathrm{w}} \leqslant-\sqrt{6}$. Obviously, within the overlapping range $f_{\mathrm{w}} \leqslant-\sqrt{6}$, the FBL and BBL profiles differ from

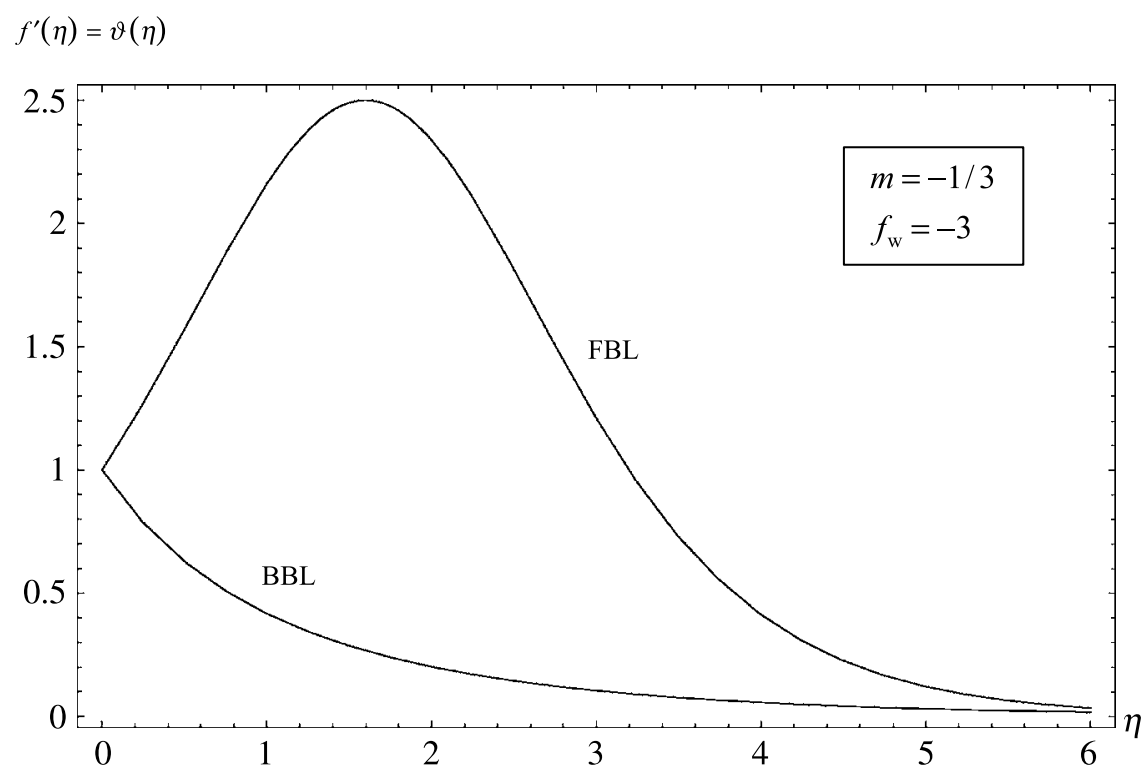

Figure 5. Dimensionless velocity and temperature profiles $f^{\prime}(\eta)=\vartheta(\eta)$ of the FBL and BBL solutions, respectively, corresponding to $m=-1 / 3, f_{\mathrm{W}}=-3$. The heat transfer coefficient has the same value $H\left(f_{\mathrm{W}}\right)=-s \cdot f^{\prime \prime}(0)=-1$ (reversed wall heat flow) in both of these cases, but the flow characteristics are quite different. 
each other. This is illustrated in Figure 5. While in the FBL-case the negative values of $f_{\mathrm{w}}$ correspond to a lateral injection, in the BBL-case the values $f_{\mathrm{w}} \leqslant-\sqrt{6}$ are associated with a lateral suction of the fluid.

\section{2. $\operatorname{CASE} c_{1} \neq 0$}

In this case, in contrast to Equation (36), we have $H \neq f_{\mathrm{w}} / 3$. Equation (27) and the third boundary condition (13) show that for $C_{1} \neq 0$ the solutions, if any, possess the algebraic asymptotic behavior

$$
f^{2}(\eta) \underset{\eta \rightarrow \infty}{\sim} 6 s C_{1} \eta, \quad C_{1} \neq 0
$$

which obviously implies $\operatorname{sgn} C_{1}=s$. The heat transfer coefficient (15) in this case is

$$
H=\frac{1}{3} f_{\mathrm{w}}-s C_{1} \leqslant \frac{1}{3} f_{\mathrm{w}} .
$$

With the aid of the transformation

$$
f(\eta)=\left(36 C_{1}\right)^{1 / 3} \frac{\mathrm{d}}{\mathrm{d} z}[\ln W(z)], \quad z=\left(\frac{s C_{1}}{6}\right)^{1 / 3}\left(\eta+\frac{C_{2}}{C_{1}}\right)
$$

we obtain for the new dependent variable $W=W(z)$ Airy's equation $W^{\prime \prime}=$ $z W$ (where the primes denote differentiation with respect to the new independent variable $z$ ). In this way, the general solution of Equation (27) for $C_{1} \neq 0$ is

$$
f(\eta)=\left(36 C_{1}\right)^{1 / 3} \frac{A i^{\prime}(z)+b B i^{\prime}(z)}{A i(z)+b B i(z)}
$$

where $A i(z)$ and $B i(z)$ denote the Airy functions (Abramowitz and Stegun, 1966) and $b$ is an integration constant.

Bearing in mind the asymptotic behavior of the Airy functions (Abramowitz and Stegun, 1966), it is easy to show that the function (40) satisfies the asymptotic condition (37) for any value of the integration constant $b$. We are left in this way with two integration constants, $C_{1}$ and $b$ and a single effective boundary condition $f(0)=f_{\mathrm{w}}$ which requires

$$
b \equiv b\left(f_{\mathrm{w}}, H\right)=-\frac{f_{\mathrm{w}} \cdot z_{0}^{1 / 2} \cdot A i\left(z_{0}\right)-\left(6 s+f_{\mathrm{w}}^{2}\right)^{1 / 2} \cdot A i^{\prime}\left(z_{0}\right)}{f_{\mathrm{w}} \cdot z_{0}^{1 / 2} \cdot B i\left(z_{0}\right)-\left(6 s+f_{\mathrm{w}}^{2}\right)^{1 / 2} \cdot B i^{\prime}\left(z_{0}\right)}
$$

where

$$
z_{0}=\left.z\right|_{\eta=0}=\left(6 s+f_{\mathrm{w}}^{2}\right) \cdot\left(36 C_{1}\right)^{-2 / 3}, \quad C_{1}=s \cdot\left(\frac{1}{3} f_{\mathrm{w}}-H\right) .
$$

Now, for a given value of $f_{\mathrm{w}}$ (and $s=+1$ or -1 ) we have to seek such solutions $\left(b, C_{1}\right)$ of Equation (41) which satisfy the condition $\operatorname{sgn} C_{1}=s$ and lead to physical solutions of our boundary value problem (i.e. to solutions $f^{\prime}(\eta)$ which 
are bounded and positive functions of $\eta$ for any $0 \leqslant \eta<\infty$, as mentioned above). It is easy to show that the entrainment/displacement velocities of the corresponding flows are always zero, $v(x, \infty)=0$.

Let us first discuss the BBL case $(s=-1)$ under this aspect. For $f_{\mathrm{w}}=0$ this problem has already been solved by Kuiken (1981a) in the context of BBL flows induced by continuous permeable surfaces stretching with velocity $u_{\mathrm{w}} \sim x^{-1 / 3}$. As shown by Kuiken (1981a), the problem admits in this special case a unique solution which in our notation corresponds to $b=0$ and $H=C_{1}=-0.39700$ (Kuiken's $c$ is related to our $C_{1}$ as $c=-\sqrt{2} \cdot C_{1}=0.56144$ ). Hence, in the parameter plane $\left(f_{\mathrm{w}}, H\right)$ this solution corresponds to the point $(0,-0.39700)$ and it is indicated in Figure 4 accordingly. A detailed analytical and numerical investigation shows that for $f_{\mathrm{w}} \neq 0$ (and $s=-1$ ) physical solutions exist only for $b=0$ and $f_{\mathrm{w}} \geqslant-\sqrt{6}$. The corresponding values of $C_{1}$ can be calculated from Equations (41) and (42). The heat transfer coefficient (38) obtained in this way is plotted as a function of $f_{\mathrm{w}} \geqslant-\sqrt{6}$ in Figure 4. It is worth noticing here that as $f_{\mathrm{w}} \rightarrow-\sqrt{6}$ from above, $C_{1}$ goes to zero (which implies $H \rightarrow-\sqrt{2 / 3}$ ), and the Airy-function solution (40) goes over in this case precisely into the universal BBL solution (17). If however, $f_{\mathrm{w}} \rightarrow-\sqrt{6}$ from below, the exponential BBL solution (34) also approaches the universal solution (17), as mentioned above. Therefore, $\left(f_{\mathrm{w}}, H\right)=(-\sqrt{6},-\sqrt{2 / 3})$ is a branching point where the branch of the exponential BBL solutions (34) merge with the branch of the Airy-function BBL solutions (40) having an algebraic asymptotic behavior. The result of this merger is the universal BBL solution (17). In addition, in the point $\left(f_{\mathrm{w}}, H\right)=(-\sqrt{6},-\sqrt{2 / 3})$ also the FBL solution (32) is present. It is also interesting to mention that for $f_{\mathrm{w}}=+\sqrt{6}$ (and $s=-1$ and $b=0$ ), where according to Equation (42) $z_{0}=0$, the solution of Equation (41) can even be calculated 'by hand'. It is

$$
H=\frac{\sqrt{6}}{3}\left(1+\frac{1}{2}\left[\frac{A i(0)}{A i^{\prime}(0)}\right]^{3}\right)=-0.23721
$$

and thus, the coordinates of the corresponding point of the parameter plane of Figure 4 are $\left(f_{\mathrm{w}}, H\right)=(+\sqrt{6},-0.23721)$.

Let us now turn to the FBL case $(s=+1)$ of the Airy function solutions (40). In contrast to the BBL case $(s=-1)$, Equation (40) yields physical FBL solutions also for non-vanishing values of the integration constant $b$. As shown recently by Magyari et al. (2003), for an impermeable plate, $f_{\mathrm{w}}=0$, the existence domain of physical FBL solutions is

$$
0<b<-\frac{A i^{\prime}(0)}{B i^{\prime}(0)}=\frac{1}{\sqrt{3}} \cong 0.57735 \text { and } 0<C_{1}<+\infty .
$$

The dependence of the corresponding heat transfer coefficient (38) on the parameter $b$ is shown in Figure 6. To every point of the curve of Figure 6, there corresponds a physical FBL solution of the problem. Hence, for $f_{\mathrm{w}}=0$ we obtain 


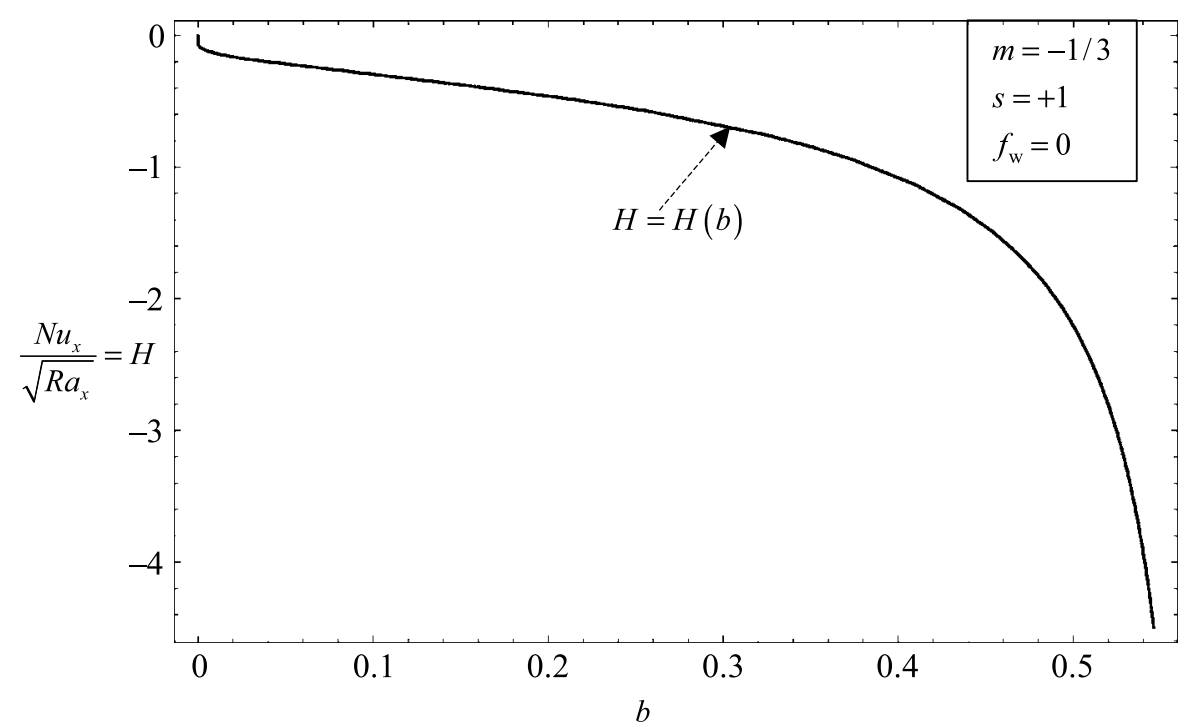

Figure 6. For $m=-1 / 3, f_{\mathrm{W}}=0$ and $0<b<-3^{-1 / 2}$, to every point of the curve $H=H(b)$ there corresponds for a physical FBL solution.

multiple FBL solutions, one for any $0<b<1 / \sqrt{3}$ and $C_{1}=-H$, where $H$ is the corresponding point of the curve of Figure 6.

When the integration constant $b$ approaches the upper limit of the interval (44), $H=-C_{1}$ goes to $-\infty$ according to

$$
H=-\frac{1}{\sqrt{2}}\left[\Gamma\left(\frac{1}{3}\right)\right]^{3 / 4}\left[3 \cdot \Gamma\left(\frac{2}{3}\right)\right]^{-3 / 4}(1-\sqrt{3} \cdot b)^{-3 / 4} .
$$

On the other hand, at the lower end of the interval (44) we obtain the behavior

$$
H=\frac{2 \sqrt{6}}{9 \ln (2 b)} \rightarrow 0^{-} \quad \text { as } \quad b \rightarrow 0^{+} .
$$

As $b$ and $H$ approach zero according to (46), the Airy function solution (40) goes over into the tanh-solution (32) taken for $f_{\mathrm{w}}=0$.

It can be shown that the multiple character of the FBL solutions as described above for $b \neq 0$ and $f_{\mathrm{w}}=0$, still persist also for permeable plates, $f_{\mathrm{w}} \neq 0$. In the latter case, however, the nature of the multiplicity is much more complex. A comprehensive investigation of this problem is still missing.

\section{Summary and Conclusions}

In this paper the steady free convection boundary layer flows from a semi-infinite vertical flat plate embedded in a saturated porous medium have been compared to each other in two situations in which the definite edge of the plate is a leading 
or a trailing edge, respectively. The former case corresponds to the familiar FBL flows ascending over an upwards projecting heated plate and the latter one to the less familiar BBL flows descending over an upwards projecting cooled plate, respectively (Figure 1(a and b)). The paper was mainly concerned with the similar flows corresponding to power law wall temperature distributions with $m=+1$ and $m=-1 / 3$, respectively, where both FBL and BBL solutions are available in an explicit analytic form.

The main results of the paper can be summarized as follows.

1. There exists a universal algebraic BBL solution, valid for any value of the power-law exponent $m$. It can, however, be realized only if a certain lateral suction of the fluid is applied. In the FBL-case no comparable universal solution is known.

2. For $m=+1$, in the range $f_{\mathrm{w}}<-2$ of the suction parameter $f_{\mathrm{w}}$, exact exponentially decaying dual BBL solutions bifurcating at $f_{\mathrm{w}}=-2$ were found. To any point of the domain of the parameter plane $\left(f_{\mathrm{w}}, H\right)$ delimited by the two branches $H=H_{ \pm}\left(f_{\mathrm{w}}\right)$ of the exponentially decaying BBL's, there also corresponds a physical solution of the boundary value problem. Among them, the universal algebraic BBL solution corresponding to $\left(f_{\mathrm{w}}, H\right)=(-\sqrt{6},-\sqrt{2 / 3})$ is also present, (Figures 2 and 3 ).

3. For $m=-1 / 3$, again both exponentially and algebraically decaying BBL solutions were found. For $f_{\mathrm{w}}<-\sqrt{6}$, the domain of existence of the exponentially decaying BBL's overlaps with that of the exponentially decaying FBL solutions, the heat transfer coefficient being given in both of these cases by $H=f_{\mathrm{w}} / 3$, (Figures 4 and 5). At $f_{\mathrm{w}}=-\sqrt{6}$, the branch of the exponentially decaying BBL's (with $f_{\mathrm{w}}<-\sqrt{6}$ ) merge with a branch of algebraically decaying BBL solutions which exist for $f_{\mathrm{w}} \geqslant-\sqrt{6}$ and can be expressed in terms of the Airy function $A i$. The result of this merger is just the universal algebraic BBL. The branch of the algebraically decaying Airy function BBL solutions $\left(f_{\mathrm{w}} \geqslant-\sqrt{6}\right)$ includes for $f_{\mathrm{w}}=0$ (impermeable plate) Kuiken's BBL induced by a continuous surface stretching with velocity $u_{\mathrm{w}}(x) \sim x^{-1 / 3}$, (Kuiken, 1981a). In the case $m=-1 / 3$, also the existence of a new class of algebraically decaying FBL solutions were reported. These are multiple solutions, and can be expressed by linear combinations of the Airy functions $A i$ and $B i$ (Figure 6).

Therefore, the exactly solvable cases discussed in the present paper show that the steady free convection BBL flows are not necessarily described by algebraically decaying and the forward ones are not necessarily described by exponentially decaying solutions of the corresponding governing equations. In both cases, an asymptotic behavior of both types of is possible. The exponentially decaying BBL's reported in this paper, however, can only be realized if a sufficiently strong lateral suction is applied. In conclusion, the circumstance that the definite edge of the semi-infinite plate is a leading or a trailing edge, can lead to substantially different 
effects on the flow and heat transfer characteristics of the corresponding FBL and BBLs, respectively.

Finally, it should not remain unnoticed that the present paper has not considered further interesting cases of free convection BBL's corresponding, for example, to the temperature exponent $m=-1 / 2$ (where, at least in the FBL-case, implicit analytic solutions are known), $m=0$ (uniform surface temperature) and $m=$ $+1 / 3$ (uniform surface heat flux), respectively.

\section{References}

Abramowitz, M. and Stegun, I. A.: 1966, Handbook of Mathematical Functions, Dover, New York.

Banks, W. H. H.: 1983, Similarity solutions of the boundary layer equations for a stretching wall, J. Méc. Théor. Appl. 2, 375-392.

Brown, S. N. and Stewartson, K.: 1965, On similarity solutions of the boundary-layer equations with algebraic decay, J. Fluid Mech. 23, 673-687.

Goldstein, S.: 1965, On backward boundary layers and flow in converging passages, J. Fluid Mech. 21, 33-45.

Khan, M. A. and Stewartson, K.: 1984, On natural convection from a cooling vertical sheet, $Q . J$. Mech. Appl. Math. 37, 325-338.

Kuiken, H. K.: 1981a, On boundary layers in fluid mechanics that decay algebraically along stretches of wall that are not vanishingly small, IMA J. Appl. Math. 27, 387-405.

Kuiken, H. K.: 1981b, A backward free-convective boundary layer, Q. J. Mech. Appl. Math. 34, 397-413.

Kuiken, H. K.: 1983, A class of backward free-convective boundary layer similarity solutions, Int. J. Heat Mass Transfer 26, 655-661.

Magyari, E. and Keller, B.: 2000a, Exact analytic solutions for free convection boundary layers on a heated vertical plate with lateral mass flux embedded in a saturated porous medium, Heat Mass Transfer 36, 109-116.

Magyari, E. and Keller, B.: 2000b, Exact solutions for self-similar boundary-layer flows induced by permeable stretching walls, Eur. J. Mech. B-Fluids 19, 109-122.

Magyari, E., Pop, I. and Keller, B.: 2003, New analytical solutions of a well-known boundary value problem in fluid mechanics, Fluid Dyn. Res. 33, 351-355.

Merkin, J. H.: 1978, On solutions of the boundary-layer equations with algebraic decay, J. Fluid Mech. 88, 309-321.

Nield, D. A. and Bejan, A.: 1999, Convection in Porous Media, 2nd edn., Springer-Verlag, New York.

Pop, I. and Ingham, D. B.: 2001, Convective Heat Transfer: Mathematical and Computational Modelling of Viscous Fluids and Porous Media, Pergamon, London. 